\title{
Estudo dos indicadores de rendimento em voleibol em função do resultado do set
}

\section{Rui MARCELINO* Isabel MESQUITA* Jaime SAMPAIO** José Cicero MORAES ${ }^{* * *}$}

\section{Resumo}

0 presente estudo pretendeu identificar possiveis indicadores de rendimento, adstritos às ações de jogo, diferenciadores do resultado obtido no set em Voleibol. A amostra consistiu em 65.949 ações realizadas em 550 sets da Liga Mundial 2005, "adulto" masculinos. A coleta de dados teve como suporte instrumental o software "Volleyball Information System". Para testar as diferenças nos indicadores de rendimento, entre as equipes que vencem e perdem os sets, se utilizou o teste t de student de medidas independentes $(p<0,05)$. As observações cumpriram os requisitos de fidedignidade para serem utilizadas como ferramenta científica, tanto pela percentagem de acordos interobservador como pela estatística Kappa de Cohen. 0 presente estudo demonstrou que as equipes que vencem os sets obtêm melhores desempenhos em todas as ações de jogo (ataque, bloqueio, saque, defesa, levantamento e recepção), devido à maior frequência de ações ponto/excelente (com exceção do número de recepções excelentes) e à menor frequência de erros. As equipes que vencem os sets apresentam uma distribuição percentual dos pontos ganhos mais equilibrada entre as três ações terminais (ataque, bloqueio e saque) do que as equipes que perdem os sets. 0 ataque representa, para as equipes perdedoras, um maior peso no total de pontos ganhos através das ações terminais.

UnIteRmos: Voleibol; Análise do jogo; Indicadores de rendimento; Resultado do set.

\section{Introdução}

A análise da performance desportiva no âmbito dos Jogos Desportivos (JD), sustentada na interpretação de indicadores de rendimento, possibilita efetuar, entre outras, avaliaçôes táticas e técnicas (HuGHES, 2004) que podem ser utilizadas no planejamento dos treinamentos, com vista a incrementar o rendimento desportivo. A identificação e descodificação das estatisticas relacionadas com as ações de jogo, utilizadas como indicadores de rendimento, têm sido apresentadas na literatura com o objetivo de contribuir para a evolução dos diferentes JD (Sampaio, Janeira, Ibanez \& Lorenzo, 2006).
Nomeadamente no Voleibol, as ações de jogo emergem sequencialmente dado este JD apresentar uma estrutura externa relativamente determinista (Moutinho, 1998; Palao, Santos \& Ureña, 2004; Rocha \& Barbanti, 2004). O conhecimento desta lógica externa de desenvolvimento, que se carateriza por uma ciclicidade relativamente estável na ocorrência das diferentes açōes de jogo, é determinante para a interpretação dos diferentes indicadores de rendimento utilizados (FIGURA 1).
*Centro de Investigaçao, Formação, Inovação e Intervenção em Desporto, Faculdade de Desporto, Universidade do Porto - Portugal. **Research Center in Sports Sciences, Health and Human Development.

***Escola de Educação Física, Universidade Federal do Rio Grande do Sul. 


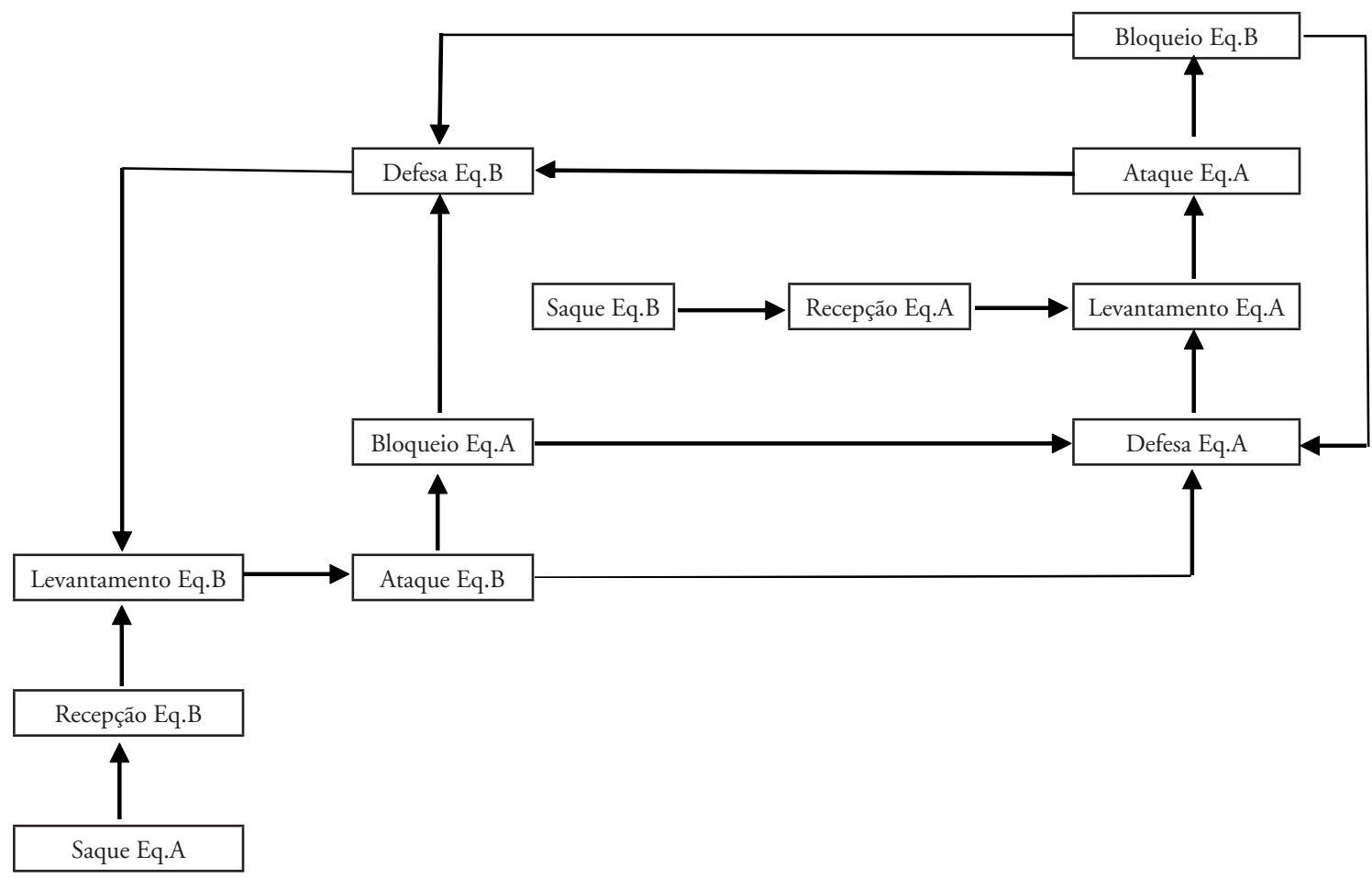

FIGURA 1 - Lógica acontecimental das ações de jogo em Voleibol.

No Voleibol, as ações de ataque, de bloqueio e de saque, devido à possibilidade de se conquistar ponto diretamente, são designadas por Ações Terminais. Por sua vez, as açóes de defesa, de levantamento e de recepção, por serem intermediárias das Ações Terminais, denominam-se de Açôes de Continuidade (Coleman, 2002).

Dos estudos realizados no Voleibol ressalta que o ataque emerge como a ação de jogo com maior poder explicativo sobre o resultado do jogo. Naquele que foi um dos primeiros estudos em que se procurou estudar a relação entre as ações de jogo de Voleibol e o resultado do set, Cox (1974) analisou 107 sets do campeonato universitário masculino dos Estados Unidos da América e concluiu que o ataque era a ação de jogo com maior poder para prever o sucesso das equipes. Por sua vez, NishiJIMA, OHSAWA e MATSUURA (1987) concluíram que o fator diferencial entre o sucesso e o insucesso no jogo de Voleibol era o "ataque após a defesa". Também Eom e Schutz (1992), com o objetivo de verificarem as características do jogo de Voleibol masculino de nível internacional, observaram 36 jogos do campeonato do mundo de 1987 e concluíram que o rendimento do ataque, em situação de contra-ataque, era o indicador de rendimento mais relacionado quer com a classificação final da competição, quer com o resultado do jogo. Mais recentemente Marcelino, Mesquita e Afonso (2008) calcularam o "ranking" das equipes, segundo os rendimentos em cada ação de jogo, e compararam com o "ranking" final da competição em análise (World Leage 2005 masculina). Concluíram que o melhor indicador de sucesso em Voleibol é o ataque, seguido pelo número de pontos no bloqueio.

Esta supremacia do ataque sobre o resultado no jogo mostra ser extensiva aos diferentes complexos de jogo, quer seja no ataque resultante da recepção do saque (complexo I), quer seja no ataque após defesa (complexo II). Zetou e Tsigilis (2007) e Zetou, Tsigilis, Moustakidis e Komninakidou (2006) observaram 38 jogos de Voleibol masculino realizados durante os Jogos Olímpicos e apresentaram os resultados em dois estudos independentes. No primeiro estudo (2006) analisaram as ações no Complexo II e concluiriam que o saque e o ataque, em situação de contra ataque, constituíam bons indicadores do rendimento desportivo. No segundo estudo (ZeTOu \& Tsigilis, 2007) centraram as suas atenções nas ações efetuadas durante o Complexo I e destacaram a recepção e o ataque como as ações mais relacionadas com o resultado do jogo.

Com exceção do estudo de Cox (1974), que utilizou o "resultado do set" como unidade de análise, todos os outros estudos referidos foram efetuados utilizando o "resultado do jogo". Sabe-se que, no Voleibol, a 
vitória no jogo ocorre quando se vencem três medidas parcelares de rendimento, i.e., quando uma equipe vence três sets. Dado que em cada set, ambas as equipes começam com zero pontos e o mesmo tem de ser ganho obrigatoriamente por uma das equipes, considera-se que um jogo de Voleibol é constituido por três, quatro ou cinco jogos quase independentes (Marcelino, Mesquita, Palao \& Sampaio, 2009).

Recentemente, a importância do estudo do set como unidade de análise do rendimento em jogo foi retomada, com destaque similar do ataque enquanto a ação de jogo com maior poder preditor sobre o resultado obtido no set. Assim, MArelic, Zufar e OMRCEN (1998) observaram 149 sets do campeonato masculino da antiga Iugoslávia e concluíram que o ataque era a ação que mais poder exercia sobre o resultado final do set. Com os mesmos objetivos MArelic, RESETAR e JANKOVIC (2004) observaram 76 sets da liga italiana A1 masculina e concluíram que a variável que influenciava, com maior projeção, o resultado final do set era o "ataque após a recepção". Com um menor peso, mas também capazes de influenciar o resultado, surgiram o "ataque em situação de contra-ataque", a "recepção do saque" e o "bloqueio".

Apesar do reconhecimento da importância da informação proveniente da investigação neste tema, quer para o domínio da prática quer para a investigação, verifica-se a ausência de estudos que se debruçam sobre a análise de indicadores de rendimento capazes de distinguirem as equipes que vencem os sets das que os perdem; nomeadamente, perceber se as equipes são melhores no ataque, por fazerem mais pontos ou por errarem menos.

\section{Métodos}

\section{Caracterização da amostra}

A amostra do presente estudo foi retirada das açôes referentes a todos os jogos da Intercontinental Round da Liga Mundial 2005, "adulto" masculinos. Foram observados 550 sets nos quais foram avaliados 14.111 ataques, 7.200 bloqueios, 12.434 saques, 8.562 defesas, 13.513 levantamentos, 10.129 recepções, perfazendo um total de 65.949 ações avaliadas.
Para além disso, a investigação centrada na análise do rendimento das equipes em competição tem vindo a recorrer, de forma sistemática, a determinados indicadores de rendimento, entre os quais de destacam as percentagens e os coeficientes de performance (Palao, Santos \& Urena, 2005). Apesar dos estudos empíricos privilegiarem o recurso às percentagens, como medida de avaliação (AGELONIDIS, 2004a, 2004b; Aurelio \& VAlLín, 2003; Garcia Maquiera \& Fernández Fraga, 2003; Oliveira, Mesquita \& Oliveira, 2005; Palao, Santos \& Ureña, 2002; Ureña Espá, Calvo Ferrer \& Lozano Pérez, 2002; Yiannis, Panagiotis, IoAnnis $\&$ AlKINOI, 2004), a mesma não permite conhecer a relação que existe entre os pontos ganhos, os pontos perdidos nem o total de tentativas. Para se obter esta informação, é necessário recorrer aos valores dos coeficientes da performance (COLEMAN, 1975), que contabilizam no seu cálculo todas as execuções efetuadas, ofertando, assim, uma informação mais qualitativa, logo mais completa, do rendimento desportivo. Recentemente estes indicadores de rendimento têm surgido na literatura específica da modalidade com maior frequência providenciando informação mais substantiva sobre o rendimento das equipes em competição (MARCELINO \& MESQUITA, 2008; Marcelino, Mesquita, Castro \& Sampaio, 2008; Marcelino, Mesquita, Sampaio \& Anguera, 2009; MarCelino et al., 2009a).

Assim, constitui objetivo do presente estudo identificar possíveis indicadores de rendimento, adstritos às ações de jogo, diferenciadores do resultado obtido no set em Voleibol.

\section{Procedimentos de coleta de dados}

A coleta de dados foi efetuada sob a responsabilidade da Federação Internacional de Voleibol (FIVB) e teve como suporte instrumental o "software" "Volleyball Information System” [VIS] (FIVB, 2000) versão 1.95 de 14 Março 2002.

\section{Variáveis da análise}

A variável independente consistiu no Resultado do Set (vitória vs. derrota)

As variáveis dependentes foram agrupadas em três categorias: 
Ações do jogo (QUADRO 1)

QUADRO 1-Variáveis dependentes relativas às "ações de jogo".

\begin{tabular}{|c|c|}
\hline $\begin{array}{l}\text { Pontos/ } \\
\text { Excelente }\end{array}$ & $\begin{array}{l}\text { Número de pontos de ataque } \\
\text { Número de pontos de bloqueio } \\
\text { Número de pontos de saque } \\
\text { Número de defesas excelentes } \\
\text { Número de levantamentos excelentes } \\
\text { Número de recepções excelentes }\end{array}$ \\
\hline Erros & $\begin{array}{l}\text { Número de erros de ataque } \\
\text { Número de erros de bloqueio } \\
\text { Número de erros de saque } \\
\text { Número de erros de defesa } \\
\text { Número de erros de levantamento } \\
\text { Número de erros de recepção }\end{array}$ \\
\hline Continuidade & $\begin{array}{l}\text { Número de ataques com continuidade } \\
\text { Número de bloqueios com continuidade } \\
\text { Número de saques com continuidade } \\
\text { Número de defesas com continuidade } \\
\text { Número de levantamentos com continuidade } \\
\text { Número de recepçôes com continuidade }\end{array}$ \\
\hline
\end{tabular}

Coeficientes da performance

Utilizou-se o Coeficiente da performance proposto por Coleman (2002), que pondera as ações segundo a qualidade de execução. Assim, para o ataque, saque e bloqueio utilizou-se a fórmula apresentada na Equação 1 e para a defesa, levantamento e recepção utilizou-se a fórmula apresentada na Equação 2.

Equação 1: Coeficiente da performance para ataque, saque e bloqueio

Coef_ $=\underline{4 \times \text { açôes pontos }+2 \times \text { açōes continuidade }+0 \times \text { açôes erro }}$

Total de ações (pontos+continuidade+erro)

Equação 2: Coeficiente da performance para a defesa, levantamento e recepção

Coef $=\underline{3 \mathrm{x} \text { açôes excelentes }+1,5 \mathrm{x} \text { açōes continuidade }+0 \mathrm{x} \text { açōes erro }}$ Total de açôes (excelentes+continuidade+erro)

\section{Percentagens}

Calcularam-se as percentagens de pontos/ excelente, as percentagens de erros e as percentagens de continuidade de todas as ações, relativamente ao total de execuçōes de cada uma delas. Simultaneamente calcularam-se as percentagens de pontos de ataque, de bloqueio e de saque, relativamente ao total de pontos ganhos pela equipe (QUADRO 2).

QUADRO 2 - Variáveis dependentes relativas às "percentagens".

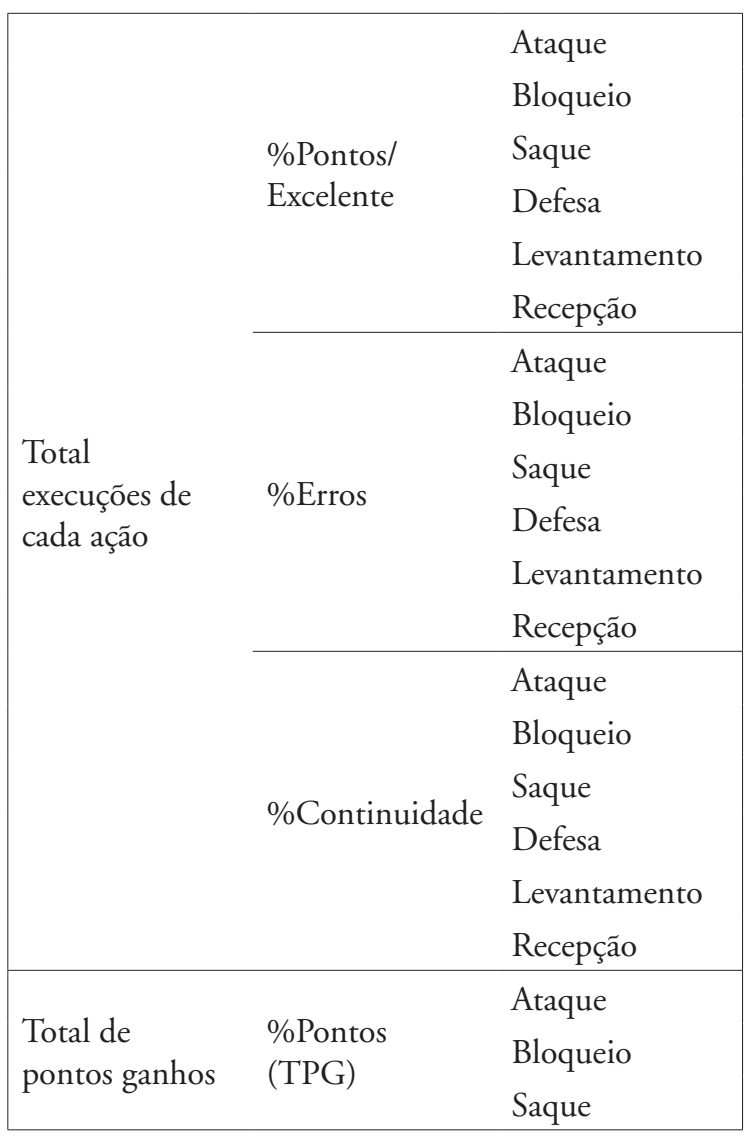

\section{Procedimentos estatísticos}

Para tratamento dos dados foi utilizada a média e o desvio padrão das variáveis dependentes segundo o Resultado do Set. Para testar a existência de diferenças entre as médias utilizou-se o teste $\mathrm{t}$ de student de medidas independentes. O nível de significância foi de $5 \%$.

\section{Fidedignidade}

Um observador independente do VIS, observou 34 sets, correspondentes a $12,36 \%$ do total de sets analisados. A qualidade dos dados observados e registados foi comprovada com a elevada percentagem de acordos interobservador (acima de 80\%) (VAN Der MARs, 1989) e pelos valores de kappa (superiores a 0,75) (BAKEMAN \& GOTTMAN, 1989) observados em todas as variáveis. 


\section{Resultados}

\section{Ações de jogo}

As equipes que vencem os sets conquistam mais pontos de ataque $(13,20 \pm 2,90)$, de bloqueio $(2,99$ $\pm 1,65)$ e de saque $(1,32 \pm 1,16)$ do que as equipes que os perdem $(11,40 \pm 3,27 ; 1,73 \pm 1,35 ; 0,79 \pm$ 0,96 , respectivamente). É também nas equipes que vencem os sets que se observa um maior número de levantamentos excelentes $(6,43 \pm 4,79$ vs. 5,29 $\pm 4,18)$ sendo, contudo, o número de recepçôes excelentes inferior $(9,37 \pm 4,36$ vs. $10,13 \pm 4,46)$. As equipes que vencem os sets alcançam um menor número de erros de ataque $(3,32 \pm 1,85)$, de bloqueio $(5,13 \pm 2,71)$, de defesa $(5,15 \pm 3,10)$ e de recepção $(0,79 \pm 1,01)$ do que as equipes que os perdem $(5,28 \pm 1,98 ; 5,72 \pm 2,62 ; 6,28 \pm 3,66$; $1,45 \pm 1,27$, respectivamente). Estas diferenças são apresentadas na FIGURA 2.

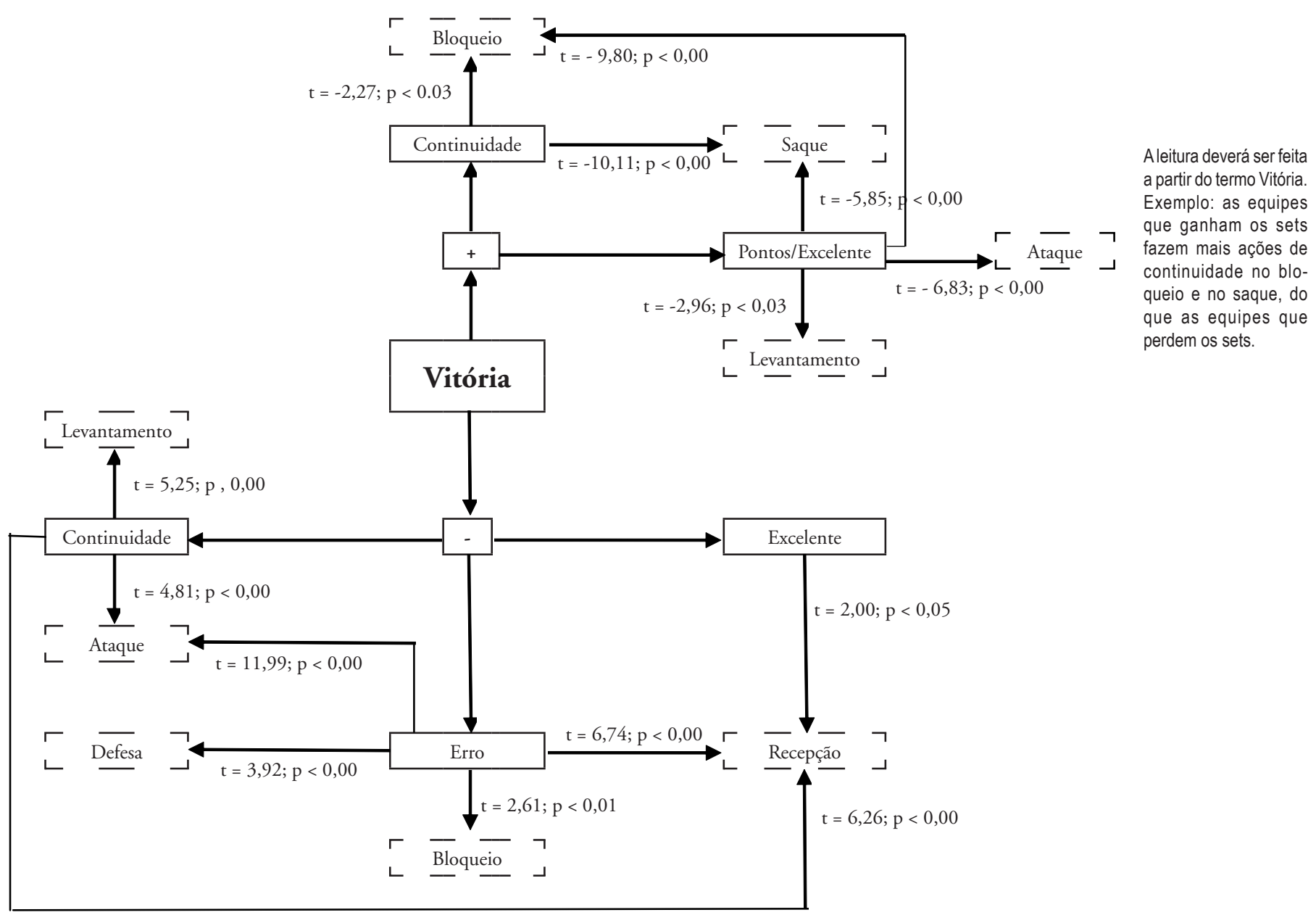

FIGURA 2 - Diferenças significativas nas ações do jogo entre as equipes que vencem os sets e as que os perdem. 


\section{Coeficientes da performance}

Na TABELA 1 são apresentados os valores dos coeficientes da performance para todas as ações de jogo.
Observam-se diferenças significativas entre as equipes que vencem os sets e as que os perdem em todos os valores dos coeficientes da performance, sendo que as primeiras apresentam sempre valores superiores às segundas.

TABELA 1 - Diferenças entre as médias dos coeficientes da performance de todas as ações de jogo, das equipes vencedoras e das equipes perdedoras.

\begin{tabular}{|c|c|c|c|c|c|}
\hline & Resultado do set & Média & Desvio padrão & $\mathbf{t}$ & $\mathbf{p}$ \\
\hline \multirow{2}{*}{ Coeficiente de ataque } & Derrota & 2,46 & 0,29 & \multirow{2}{*}{ - 14,916 } & \multirow{2}{*}{0,000} \\
\hline & Vitória & 2,81 & 0,27 & & \\
\hline \multirow{2}{*}{ Coeficiente de bloqueio } & Derrota & 1,48 & 0,45 & \multirow{2}{*}{$-4,564$} & \multirow{2}{*}{0,000} \\
\hline & Vitória & 1,66 & 0,47 & & \\
\hline \multirow{2}{*}{ Coeficiente de saque } & Derrota & 1,68 & 0,18 & \multirow{2}{*}{$-6,697$} & \multirow{2}{*}{0,000} \\
\hline & Vitória & 1,77 & 0,17 & & \\
\hline \multirow{2}{*}{ Coeficiente de defesa } & Derrota & 1,69 & 0,66 & \multirow{2}{*}{$-3,505$} & \multirow{2}{*}{0,000} \\
\hline & Vitória & 1,89 & 0,69 & & \\
\hline \multirow{2}{*}{ Coeficiente de levantamento } & Derrota & 2,25 & 0,41 & \multirow{2}{*}{$-3,601$} & \multirow{2}{*}{0,000} \\
\hline & Vitória & 2,39 & 0,49 & & \\
\hline \multirow{2}{*}{ Coeficiente de recepção } & Derrota & 2,38 & 0,55 & \multirow{2}{*}{$-4,542$} & \multirow{2}{*}{0,000} \\
\hline & Vitória & 2,59 & 0,56 & & \\
\hline
\end{tabular}

\section{Percentagens}

A observação da FIGURA 3, onde se apresentam as diferenças significativas de todas as percentagens analisadas segundo o resultado no set, deixa evidente que são as equipes que vençam os sets aquelas que obtêm percentagens superiores de ações ponto/ excelentes. Esta superioridade observa-se em todas as ações de jogo.

Contudo, e no que se refere à percentagem de pontos relativa ao total de pontos ganhos do ataque, observa-se, curiosamente, que as equipes que vencem os sets obtêm uma percentagem inferior de pontos de ataque. Em suma, de todos os pontos que ganham, as equipes vencedoras conquistam percentualmente mais pontos de bloqueio e de saque e menos pontos de ataque. Por seu turno, a percentagem de erros é inferior nas equipes que vencem os sets para as açóes de ataque, bloqueio, saque, defesa e recepção, sendo que apenas não se observam diferenças na percentagem de erros de levantamento. 


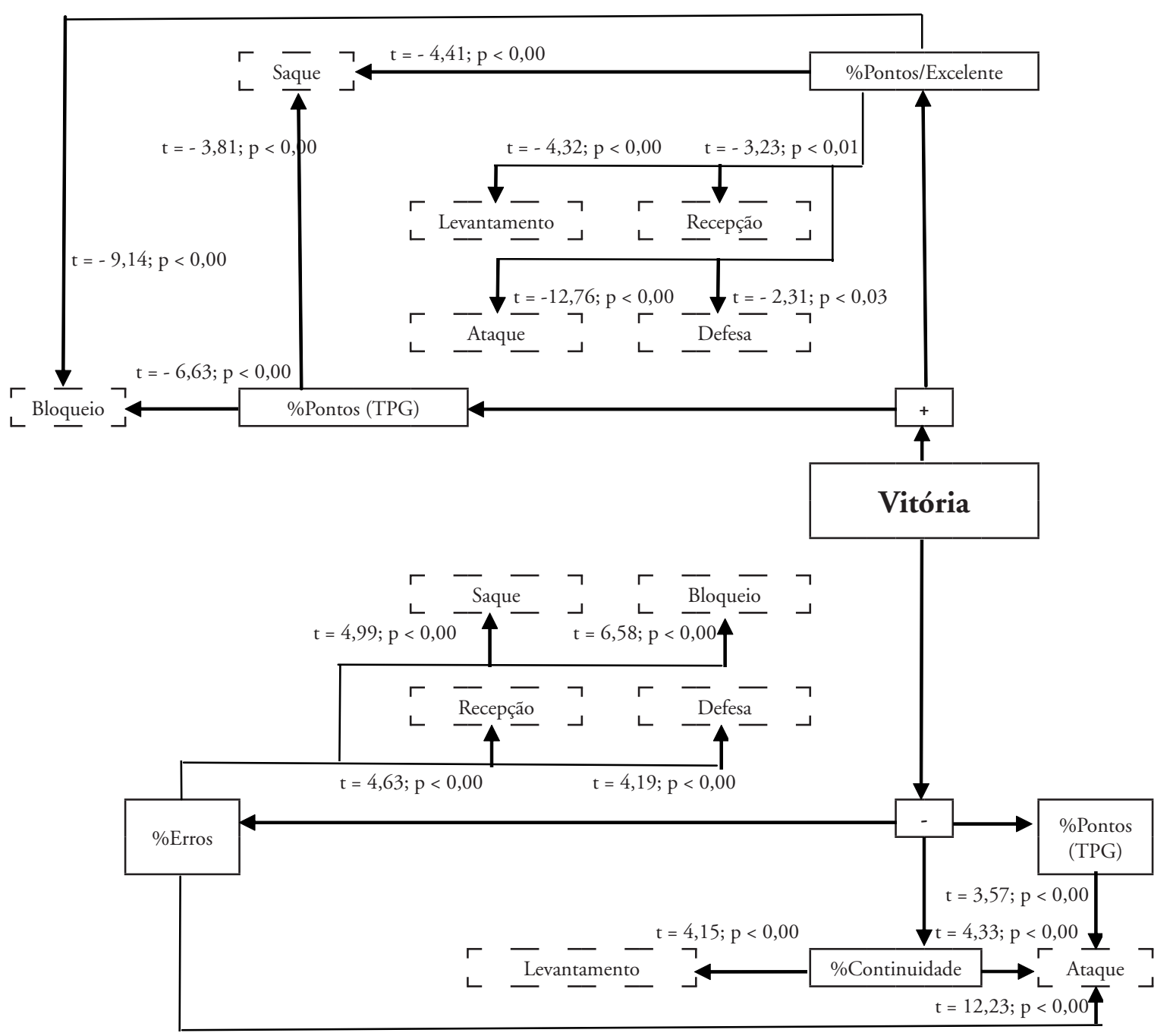

Aleitura deverá ser feita a partir do termo Vitória. Exemplo: as equipes que ganham os sets fazem uma menor percentagem de pontos (TPG) no ataque, do que as equipes que perdem os sets.

FIGURA 3 -Diferenças significativas nas percentagens entre as equipes que vencem os sets e as que os perdem.

\section{Discussão}

O objetivo deste estudo foi identificar possíveis indicadores de rendimento, adstritos às ações de jogo, diferenciadores do resultado obtido no set em Voleibol. Desta forma, e partindo do pressuposto que as equipes que vencem um set obtêm rendimentos superiores, em determinados indicadores, pretendeuse identificar quais as açôes de jogo que assumem um papel decisivo para o desfecho final dos sets.

Os resultados demonstram que para se obter uma vitória no set é necessário alcançar coeficientes da performance superiores na totalidade das açōes de jogo. Na literatura disponível apenas é realçada a importância do ataque (Marcelino, MesQuita, Castro \& Sampaio, 2008; Marelic, Resetar \& JanKovic, 2004; Rocha \& BarbanTI, 2004), do bloqueio (EOM \& SCHUTZ, 1992; Marcelino et al., 2008; Marelic, RešEtar, ZadražNik
\& ĐurKovic, 2005), do saque (Zetou et al., 2006) e da recepção (Marelic, Resetar \& Jankovic, 2004; Zetou \& TsigiLIs, 2007). É possível verificar que os estudos identificados na literatura não evidenciam que as equipes que vencem os sets alcançam rendimentos superiores nas ações de levantamento e de defesa. No presente estudo, os valores dos coeficientes, demonstram existirem diferenças significativas tanto para o levantamento $(\mathrm{t}=$ $-3,60 ; \mathrm{p}=0,00)$ como para a defesa $(\mathrm{t}=-3,01 ; \mathrm{p}=0,00)$ entre as equipes que vencem os sets e as que os perdem, com vantagem para as primeiras.

Verifica-se ainda que na recepção as equipes vencedoras fazem menos recepçôes excelentes embora também errem menos, conseguindo obter uma relação entre ambas mais favorável, materializada na obtenção de valores superiores do coeficiente da performance. Mais 
se acrescenta que o fato de as equipes que concretizam menos recepçõos excelentes conseguirem, mesmo assim, obter rendimentos desportivos superiores, manifestados no resultado do set, atesta a mudança no conceito de "recepção excelente" no Voleibol atual. No instrumento utilizado no presente estudo, para se considerar "recepção excelente", a bola teria de ser enviada para um espaço reduzido, situado entre a zona 2 e 3 do terreno de jogo, zona ideal tradicional, sendo considerado que apenas deste local são geradas, à partida, todas as opções de ataque. No entanto, com a evolução do jogo é notório que, cada vez mais, os levantadores utilizam um espaço de intervenção mais abrangente, de forma a incutir maior dinâmica ofensiva. Um estudo realizado por EsteVES e MESQUTTA (2007), no Voleibol masculino de alto rendimento, evidenciou que a utilização de uma zona de distribuição distinta, de dimensão superior à zona ideal tradicional, permitiu todas as opções de ataque; este resultado confirma a assunção de que no Voleibol atual o espaço utilizado para a organização do jogo ofensivo tende a ser cada vez mais amplo, de forma a provocar desequilíbrios espaciais e temporais entre atacantes e bloqueadores, com vantagens para os primeiros.

A inferioridade da percentagem de pontos de ataque, relativa ao total de pontos ganhos, observada nas equipes vencedoras, denuncia um maior contributo do bloqueio e do saque para a conquista de pontos, evidenciando a maior capacidade destas equipes em utilizar estes procedimentos como ações ofensivas. De fato, as equipes que vencem os sets realizam em média 1,32 pontos de saque por set, face a 0,79 das equipes perdedoras, o que mostra o seu pendor ofensivo no jogo de alto rendimento masculino da atualidade. Esta evidência confirma-se também ao nível do bloqueio, porquanto as equipes que vencem os sets possuem maior capacidade de pontuar através desta açáo de jogo.

Os resultados apresentados sugerem que o sucesso no Voleibol de alto nível, na atualidade, exige das equipes uma elevada estabilidade e equilíbrio no rendimento das diferentes açōes de jogo, sendo desta relação de compromisso que se geram condições propícias para vencer o set. Esta necessidade é confirmada no fato das equipes perdedoras contarem com mais pontos ganhos através do ataque, e com um menor número de pontos ganhos pelo saque e pelo bloqueio do que as equipas que alcançam sucesso. Tal resultado evidencia a importancia da treinabilidade sustentada de todas as ações de jogo, não previligiando em demasia uma delas, como seja $o$ ataque. A necessidade deste equilibrio é confirmado pelo fato de não se observar uma supermacia das equipes com sucesso apenas no ataque, mas também nas restantes ações terminais (saque e bloqueio).

\title{
Conclusão
}

Os resultados do presente estudo permitem concluir que no Voleibol masculino, de elevado rendimento desportivo, os desempenhos obtidos ao nível das ações de jogo (ataque, bloqueio, saque, defesa, levantamento e recepção) distinguem significativamente as equipes que vencem os sets das que os perdem. A superioridade das equipes vencedoras é confirmada na maior frequência de ações ponto/excelente (com exceção do número de recepções excelentes) e na menor frequência de erros. Mais ainda, as equipes que vencem os sets apresentam uma distribuição percentual dos pontos ganhos mais equilibrada entre as três ações terminais (ataque, bloqueio e saque), do que as equipes que perdem os sets. Daí resulta que o ataque representa para as equipes perdedoras, um maior "peso" no total de pontos ganhos através das ações terminais.

\begin{abstract}
Study of performance indicators in male volleyball according to the set results

The present study aimed to identify in which performance indicators are there differences between the teams that win and lose the sets in Volleyball matches. Archival data were obtained from 65.949 game actions in 550 sets from the men's 2005 World League. The "Volleyball Information System" was used for data gathering. Descriptive statistics were performed on all variables and the student $t$-test was used. Kappa of Cohen analysis and percentage of agreement demonstrated good inter-observer reliability
\end{abstract}


which confirmed the accuracy of observations. This study showed that the teams that win their sets have best performance in all game actions (attack, block, serve, dig, set, reception) due to higher frequency of point/excellent actions (with the exception of the number of excellent receptions) and a lower frequency of errors. The teams that win those sets have a more balanced distribution of points earned between the three terminal actions (attack, block and service) than the teams that lose their sets. For the losing teams, the attack represents a greater weight in the total points earned through the terminal actions.

UNITERMS: Volleyball; Notational analysis; Performance indicators; Set results.

\section{Referências}

AGELONIDIS, Y. Fluctuations of different aspects of group cohesion across a competitive season in volleyball teams. Journal of Human Movement Studies, London, v.46, p.459-71, 2004a.

The jump serve in volleyball: from oblivion to dominance. Journal of Human Movement Studies, London, v.47, p.205-13, $2004 b$.

AURELIO, J.; VALLÍN, D. Diferencias entre el voleibol de alto nivel masculino y femenino: análisis para un rendimiento óptimo. Voley Total: Revista oficial de la Real Federación Española de Voleibol, Madrid, v.1, p.34-9, 2003.

BAKEMAN, R.; GOTTMAN, J.M. Observación dela interacción: introducción al análisis secuencial. Madrid: Ediciones Morata, 1989. COLEMAN, J. A statistical evaluation of selected volleyball techniques at the 1974 World's Volleyball Championships. (Unpublished doctoral dissertation) - Brigham Young University, Provo, 1975.

Scouting opponents and evaluating team performance. In: SHONDELL, D. (Ed.). The volleyball coaching bible. Champaing: Human Kinetics, 2002. p.321-46.

COX, R.H. Relationship between selected volleyball skill components and team performance of men's northwest 'AA' volleyball teams. Research Quarterly of the American Association for Health, Physical Education and Recreation, Reston, v.45, n.4, p.441-6, 1974.

EOM, H.J.; SCHUTZ, R.W. Statistical analyses of volleyball team performance. Research Quarterly for Exercise and Sport, Washington, v.63, n.1, p.11-8, 1992.

ESTEVES, F; MESQUITA, I. Estudo da zona de distribuição no voleibol de elite masculino em função do jogador distribuidor e do tipo de passe. Revista Portuguesa de Ciências do Desporto, Porto, v.7, n.1, p.36, 2007. (Proceedings do 1.Congresso Internacional de Jogos Desportivos - Olhares e contextos da performance: da iniciação ao Rendimento). FÉDÉRATION INTERNATIONALE DE VOLLEYBALL (FIVB). VIS (Volleyball Information System) staff guidelines: evaluation criteria 2000. Lousanne: FIVB, 2000.

GARCIA MAQUIERA, F.A.; FERNÁNDEZ FRAGA, F. Acciones determinantes en el resultado en voleibol. Vigo: Real Federación Española de Voleibol, 2003. (Congreso Internacional sobre Entrenamiento Deportivo: "Promocón y tecnificación. Instrumentos para el desarrollo del Voleibol, 2003). Disponível em: <http://fmvolei.org.br/arquivos/accionesDeterminantes.pdf s. HUGHES, M. Performance analysis: a 2004 perspective. International Journal of Performance Analysis in Sport, Cardiff, v.4, p.103-9, 2004.

MARCELINO, R.; MESQUITA, I. Associations between performance indicators and set's result on male volleyball. In: INTERNATIONAL SCIENTIFIC CONFERENCE ON KINESIOLOGY, 5., 2008, Zagreb. Proceedings.... Zagreb: University of Zagreb, 2008. p.955-7.

MARCELINO, R.; MESQUITA, I.; AFONSO, J. The weight of terminal actions in volleyball: contributions of the spike, serve and block for the teams' rankings in the World League'2005. International Journal of Performance Analysis in Sport, Cardiff, v.8, n.2, p.1-7, 2008.

MARCELINO, R.; MESQUITA, I.; CASTRO, J.; SAMPAIO, J. Sequential analysis in volleyball attack performance: a log-linear analysis. Journal of Sport Science, London, v.26, p.S83-S84, 2008. Supplement 2.

MARCELINO, R.; MESQUITA, I.; PALAO, J.; SAMPAIO, J. Home advantage in high-level volleyball varies according to set number. Journal of Sports Science and Medicine, Belconnen, v.8, p.352-6, 2009a.

MARCELINO, R.; MESQUITA, I.; SAMPAIO, J.; ANGUERA, M. Home advantage in high-level volleyball [Ventaja de jogar en casa en Voleibol de alto rendimiento]. Revista de Psicologia del Deporte, Palma de Malorca, v.18, n.2, p.181-96, 2009 b. 
MARELIC, N.; RESETAR, T.; JANKOVIC, V. Discriminant analysis of the sets won and the sets lost by one team in A1 Italian volleyball league-a case study. Kinesiology, Zagreb, v.36, n.1, p.75-82, 2004.

MARELIC, N.; REŠETAR, T.; ZADRAŽNIK, M.; ĐURKOVIC, T. Modelling of situation parameters in top level volleyball. In: INTERNATIONAL SCIENTIFIC CONFERENCE ON KINESIOLOGY, 4., 2005, Opatija. Proceedings.... Opatija: [s.ed.], 2005. p.459-64.

MARELIC, N.; ZUFAR, G.; OMRCEN, D. Influence of some situation-related parameters on the score in volleyball. Kinesiology, Zagreb, v.30, n.2, p.55-65, 1998.

MOUTINHO, C. O ensino do voleibol: a estrutura funcional do voleibol. In: GRAÇA, A.; OLIVEIRA, J. (Eds.). O ensino dos jogos desportivos. 3. ed. Porto: Centro de Estudos dos Jogos Desportivos/Faculdade do Desporto e de Educação Física da Universidade do Porto, 1998. p.137-52.

NISHIJIMA, T.; OHSAWA, S.; MATSUURA, Y. The relationship between the game performance and group skill in volleyball. International Journal of Physical Education, Schorndorf, v.24, n.4, p.20-6, 1987.

OLIVEIRA, R.; MESQUITA, I.; OLIVEIRA, M. Caracterização da eficácia do ataque no voleibol de elevado rendimento competitivo: estudo aplicado em equipas masculinas participantes na Liga Mundial 2003. In: PINTO, J. (Ed.). Estudos 5. Porto: CEJD/FCDEF-UP, 2005. p.156-66.

PALAO, J.M.; SANTOS, J.A.; UREÑA, A. Incidencia del rendimiento de los complejos de juego por rotaciones sobre la clasificación final de los JJOO de Sydney 2000. Valladolid: Real Federación Española de Voleibol, 2002. (8. Congreso Internacional sobre Entrenamiento Deportivo: "Tendencias actuales en el Voleibol mundial de máxino nivel”, 2002). Disponível em: <http://www.fmvolei.org.br/arquivos/incidenciaRendimento.pdf >.

Effect of team level on skill performance in volleyball. International Journal of Performance Analysis in Sport, Cardiff, v.4, p.50-60, 2004.

The effect of the setter's position on the spike in volleyball. Journal of Human Movement Studies, London, v.48, p.25-40, 2005.

PARLEBAS, P. Analyse et modélation du volley-ball de haute compétition. Science et Motricité, Ronchin, v.4, p.3-22, 1988. ROCHA, C.; BARBANTI, V. Uma análise dos fatores que influenciam o ataque no voleibol masculino de alto nível. Revista Brasileira Educação Física Esporte, São Paulo, v. 8, n.4, p.303-14, 2004.

SAMPAIO, J.; JANEIRA, M.; IBANEZ, S.; LORENZO, A. Discriminant analysis of game-related statistics between basketball guards, forwards and centres in three professional leagues. European Journal of Sport Science, Birmingham, v.6 n.3, p.173-8, 2006. UREÑA, A.; CALVO, R.; LOZANO, C. Estudio de la recepcion del saque en el Voleibol masculino español de elite tras la incorporacion del jugador libero. Revista Internacional de Medicina y Ciencias de la Actividad Física y Deporte, Granada, v.2, p.39-49, 2002. VAN DER MARS, H. Observer reliability: issues and procedures. In: DARST, P.W.; ZAKRAJSEK, D.B.; MACINI, V.H. (Eds.). Analyzing physical education and sport instruction. Champaign: Human Kinetics, 1989. p.53-79.

YIANNIS, L.; PANAGIOTIS, K.; IOANNIS, A.; ALKINOI, K. A comparative study of the effectveness of Greek national men's volleyball team with internationally top-ranked teams. International Journal of Volleyball Research, Austin, v.7, n.1, p.4-9, 2004. ZETOU, E.; TSIGILIS, N. Does efectiveness os skill in complex I predict win in men's olympic volleyball games? Journal of Quantitative Analysis in Sports, Berkeley, v.3, n.4, p.1-9, 2007.

ZETOU, E.; TSIGILIS, N.; MOUSTAKIDIS, A.; KOMNINAKIDOU, A. Playing characteristics of men's Olympic Volleyball teams in complex II. International Journal of Performance Analysis in Sport, Cardiff, v.6, p.172-7, 2006.

\section{Agradecimentos}

Este estudo foi financiado pela Fundação para a Ciência e a Tecnologia - Portugal (SFRH/BD/38776/2007) e pelo Programa Operacional para a Ciência e Inovação (POCI 2010) co-financiado pelo Fundo Social Europeu (FEDER).

ENDEREÇO

Isabel Mesquita

Gabinete de Voleibol

Faculdade de Desporto

Universidade do Porto

R. Dr. Plácido Costa, 91

4200-450 - Porto - PORTUGAL

e-mail: rui_barroselas@hotmail.com
Recebido para publicação: 15/01/2008

1a. Revisão: 03/03/2009

2a. Revisão: 11/09/2009

Aceito: 23/09/2009 